tion of the rate of appearance of one or another specific cargo proteins in CSF after ${ }^{2} \mathrm{H}_{2} \mathrm{O}$ labeling could reveal neuronspecific defects that occur early in a disease process. More generally, stable isotope labeling of precursor pools followed by either cross-sectional or longitudinal CSF sampling offers a powerful tool to explore aspects of pathologic processes in the brain that are unlikely to be detected by either proteomic or metabolomic measures of absolute steady-state concentrations. Combining measures of total analyte and rates of appearance of labeled newly synthesized forms should increase our ability to define early stages of neurodegenerative diseases and facilitate the development and testing of novel therapies targeted to the pathologic process. In other words, treating the biomarker (e.g., cholesterol) may offer a path to prevent- ing or delaying the clinical symptoms that lie downstream of that biomarker and are often irreversible consequences of failing to intervene early.

\section{Acknowledgments}

The author thanks Julie Stone (Merck Research Laboratories) for sharing her insights on what needs to be understood to fully interpret changes in CSF concentrations of analytes.

Address correspondence to: William Z. Potter, NIMH, 6001 Executive Blvd., BG NSC RM 7209, Rockville, Maryland 20892, USA. Phone: 267.970.6604; E-mail: wzpottermd@gmail.com.

1. Fanara $P$, et al. Cerebrospinal fluid-based kinetic biomarkers of axonal transport in monitoring neurodegeneration. J Clin Invest. 2012; 122(9):3159-3169.
2. Fanara $P$, et al. In vivo measurement of microtubule dynamics using stable isotope labeling with heavy water. Effect of taxanes. J Biol Chem. 2004; 279(48):49940-49947.

3. Fanara P, et al. Stabilization of hyperdynamic microtubule is neuroprotective in ALS.J Biol Chem. 2007;282(32):23465-23472

4. Bertilsson L, Asberg M, Thoren P. Differential effect of chlorimipramine and nortriptyline on cerebrospinal fluid metabolites of serotonin and noradrenaline in depression. Eur J Clin Pharmacol. 1974; 7(5):365-368

5. Kopin IJ. Measuring turnover of neurotransmitters in human brain. In: Lipton MA, DiMascio A, Killam KF, eds. Psychopharmacology: A Generation of Progress. New York, New York, USA: Raven Press; 1978:933-942.

6. Hu WT, et al. Novel CSF biomarkers of Alzheimer's disease and mild cognitive impairment. Acta Neuropathol. 2010;119(6):669-678.

7. Zlokovic BV. Clearing amyloid through the bloodbrain barrier. J Neurochem. 2004;89(4):807-811.

8. Pollack A. Alzheimer's drug fails its first big clinical trial. New York Times. July 24, 2012:B4.

9. Bateman RJ, et al. A $\gamma$-secretase inhibitor decreases amyloid- $\beta$ production in the central nervous system. Ann Neurol. 2009;66(1):48-54

\title{
When NK cells overcome their lack of education
}

\author{
Baptiste N. Jaeger ${ }^{1,2,3}$ and Eric Vivier ${ }^{1,2,3,4}$
}

${ }^{1}$ Centre d'Immunologie de Marseille-Luminy, Aix-Marseille Université UM2, Campus de Luminy case 906, Marseille, France. ${ }^{2}$ INSERM U1104, Marseille, France. ${ }^{3}$ CNRS, UMR7280, Marseille, France. ${ }^{4}$ Assistance Publique — Hôpitaux de Marseille, Hôpital de la Conception, Marseille, France.

\begin{abstract}
Cells of the immune system have evolved various molecular mechanisms to sense their environment and react to alterations of self. NK cells are lymphocytes with effector and regulatory functions, which are remarkably adaptable to changes in self. In a study published in this issue of the JCI, Tarek and colleagues report the clinical benefits of manipulating NK cell adaptation to self in an innovative $\mathrm{mAb}$-based therapy against neuroblastoma (NB). This novel therapeutic strategy should stimulate further research on NK cell therapies.
\end{abstract}

NK cells are involved in the elimination of tumor cells and infected cells (1); they can kill their cellular targets via cytotoxic granule exocytosis and also secrete cytokines such as IFN- $\gamma$ that participate in the shaping of the adaptive immune response. NK cells express a wide range of surface molecules that include inhibitory and activating receptors; in humans, this family comprises the natural cytotoxicity receptors (NKp30, NKp44, and NKp46), the Fcy receptor IIIA (CD16), and the activating killer cell immunoglobulin-like receptors (KIRs). CD16 endows NK cells with antibody-dependent cell-mediated cytotoxicity

Conflict of interest: Eric Vivier is a cofounder and shareholder of Innate Pharma.

Citation for this article: J Clin Invest. 2012; 122(9):3053-3056. doi:10.1172/JCI63524.
(ADCC) properties. These activating receptors associate with immunoreceptor tyrosine-based activation motif-bearing adaptors to transduce potent activating signals. The inhibitory KIRs in humans (and their functional homologs in mice, the Ly49 receptors) recognize classical major histocompatibility complex class Ia molecules (MHC-I) and transduce inhibitory signals via their intracytoplasmic tyrosine-based inhibition motifs. Upon NK cell encounter with potential target cells, the integration of activating and inhibitory signals dictates the NK cell response. Normal self cells expressing high levels of MHC-I and low levels of activating ligands are spared, while stressed cells with downregulated MHC-I expression and high levels of activating ligands activate NK cells and are killed.

\section{NK cell education}

The functional maturation of NK cells includes a process of education, also referred as to licensing, arming, or tuning, by which NK cells acquire effector functions that are adapted to the host in which they develop (2-5). In addition to its wellknown role in the regulation of NK cell effector functions, MHC-I recognition by inhibitory receptors is also involved in NK cell education. When NK cells cannot sense self MHC-I, as in MHC-I-deficient mice or patients, individuals do not develop autoimmune disorders, and the NK cells are hyporesponsive to stimulation in vitro (Figure 1A) (6-8). Various conflicting models have been proposed to describe how the interaction of MHC-Ispecific receptors with their ligands contributes to NK cell education (2-5). A unifying model inspired from the arming/ disarming model initially proposed by David Raulet and colleagues (2) is consistent with the experimental data published so far. In this scenario, NK cells sense target cells via the combined engagement of activating, inhibitory, and adhesion receptors. The intensity of the NK cell response is commensurate with the inte- 
A

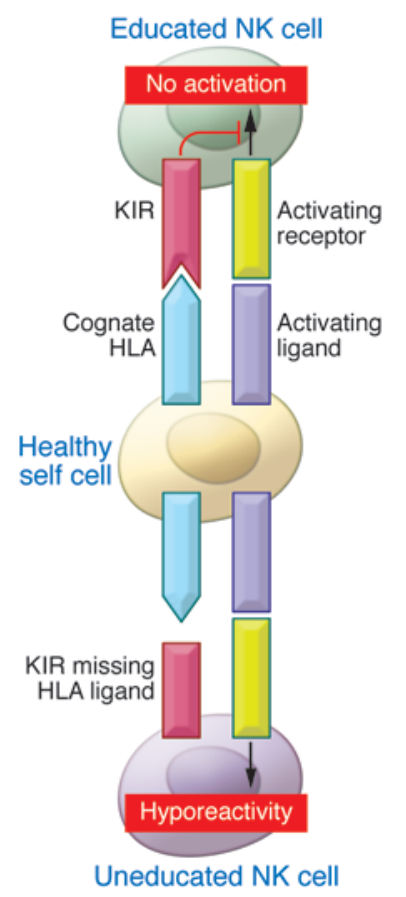

B

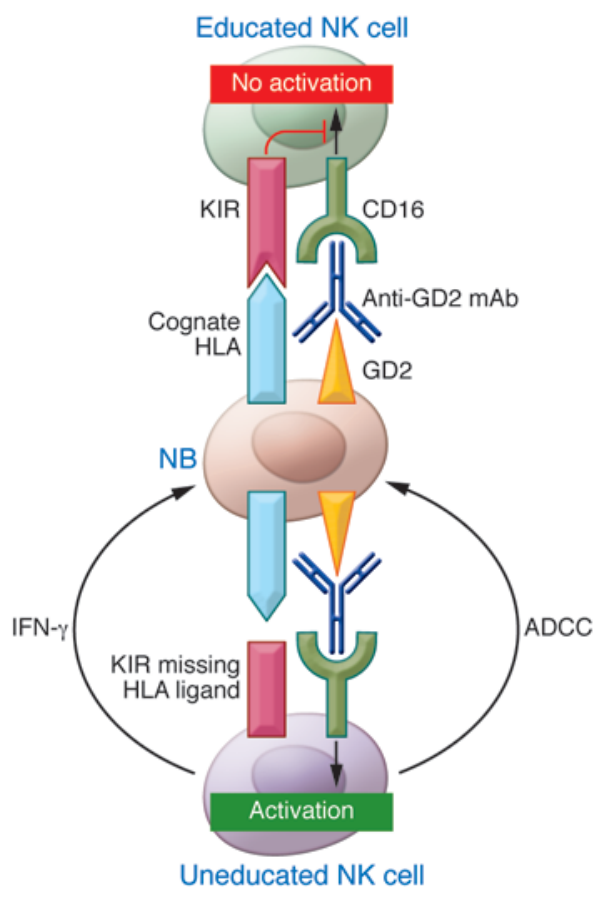

\section{Figure 1}

Activation of educated NK cells can be blocked through their inhibitory KIR receptors. (A) NK cell tolerance toward self cells is ensured by two mechanisms: MHC-I recognition by inhibitory KIR receptors that prevents activation of educated NK cells (top) and by the hyporeactivity of uneducated NK cells (bottom). (B) In the context of anti-GD2 (3F8) treatment, NB cells expressing GD2 will be decorated with this mAb. The activating receptor CD16, expressed on NK cells, recognizes 3F8, but only uneducated NK cells will mediate strong ADCC (bottom), as the engagement of inhibitory KIR receptors by their cognate HLA ligands expressed on NB blocks the activation of educated NK cells (top). gration of these signals. Upon encountering target cells expressing activating ligands but lacking MHC-I, NK cells are activated rapidly, as NK cell degranulation and cytokine production can be detected in less than 4 hours. However, the overactivation of NK cells would lead to their desensitization for further interactions with other targets. Thus, in the absence of host MHC-I, frequent interactions with cells lacking MHC-I and also expressing activating ligands would lead to NK cell desensitization, consistent with the hyporeactivity of NK cells observed in MHC-I-deficient patients and mice (9-11). According to this model, a key factor of the adaptation of NK cell reactivity to changes in their environment resides in their duration. An acute downregulation of MHC-I expression would lead to NK cell activation, whereas a chronic downregulation of MHC-I would lead to the chronic activation of NK cells and induce their hyporeactivity.

Although the exact molecular mechanisms leading to the hyporeactivity of uneducated NK cells are not fully understood, the confinement of activating receptors at the plasma membrane of NK cells is a major difference between educated and uneducated NK cells. In educated NK cells, activating receptors localize in nanodomains, while they remain linked to actin meshwork structures in uneducated NK cells (12). The fact that MHC-I-dependent education does not trigger profound and permanent modifications in the developmental program of NK cells (12) renders this process more versatile than initially thought. Adoptive transfer experiments have shown that NK cells expressing inhibitory receptors can switch from a hyporesponsive to a competent status upon recognition of the cognate MHC-I molecule, highlighting the constant adjustment of the NK cell reactivity to its surrounding environment $(13,14)$.

\section{Self-MHC-I molecules do not inhibit uneducated NK cells}

In this issue of the JCI, Tarek and colleagues have analyzed the contribution of educated and uneducated NK cells (that they refer as to licensed and unlicensed NK cells, respectively) in the killing of neuroblastoma (NB) tumors upon treatment with $3 \mathrm{~F} 8$, a mAb targeting the disialoganglioside surface antigen GD2 (15). They genotyped patients with NB and divided the cohort into two groups: those lacking any HLA ligand for inhibitory KIRs (referred to as "missing KIR ligand" patients) and those with all HLA ligands for inhibitory KIRs (referred to as "all ligands present" patients). Fourteen KIR genes clustered in chromosome $19 q 13.4$ have been described in humans, four of which encode inhibitory cell surface receptors that interact with a specific group of MHC-I molecules (Figure 2 and ref. 16). This locus is subjected to extensive genetic variation, both in the number of genes present and in the sequence of each KIR allele (16). An additional level of complexity arises from the variegated expression of KIR genes in NK cell clones, leading to a vast diversity of NK cells present within each individual. As a consequence of these mechanisms and the absence of cosegregation of KIR and HLA genes, a fraction of NK cells may lack inhibitory receptors specific for self-MHC-I in each individual. Given that the interaction between inhibitory KIRs and their HLA ligands educates NK cells to acquire their full reactivity, the fraction of NK cells in the missing KIR ligand patients that only expresses inhibitory KIRs that have no cognate HLA ligand is hyporeactive in vitro (Figure 2). In contrast, all NK cells are educated in all ligands present patients. Therefore, the overall NK cell reactivity in these patients should be greater than that in missing KIR ligand patients. However, Tarek et al. found that patients with missing KIR ligands showed improved overall and progression-free survival when treated with $3 \mathrm{~F} 8$. In vitro, both educated and uneducated NK cells were activated against NB targets by the 3F8 treatment through CD16, but educated 


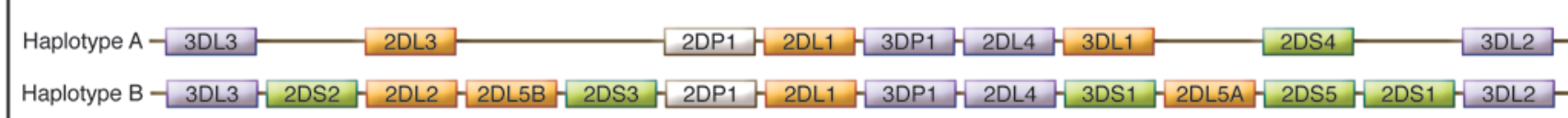

KIR haplotypic variability

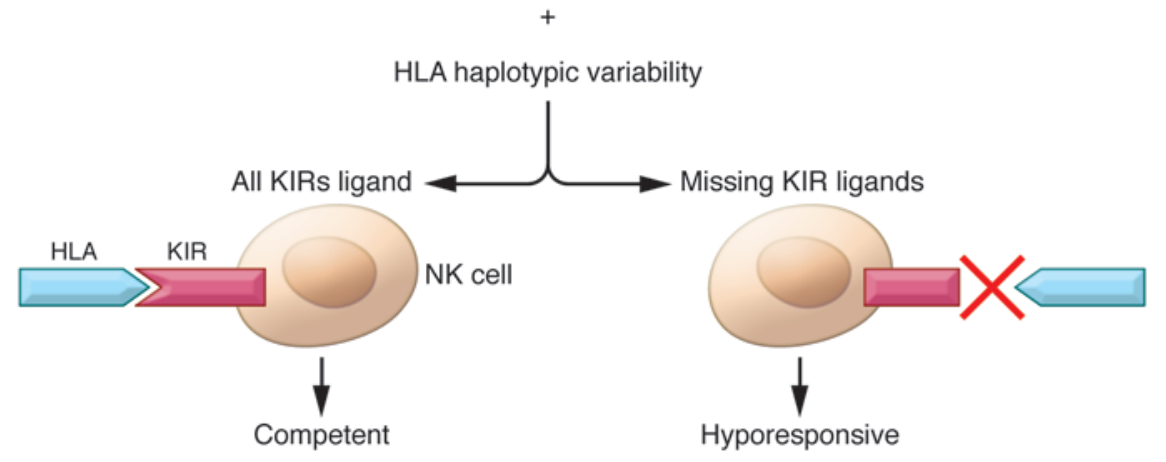

Figure 2

Some individuals express inhibitory KIR but not their HLA ligands. Schematic view of haplotypes A and B of the KIR locus representing genes encoding activating receptors (green), inhibitory receptors (orange), pseudogenes (white), and conserved genes (purple) that are pseudogenes or encode activating or inhibitory receptors. As the genes encoding the cognate HLA ligands are present on another chromosome and segregate independently of the KIRs, not all individuals express the cognate HLA ligands for their KIRs. In individuals expressing the KIRs and their HLA ligands (all KIR ligands), all NK cells are educated by the KIR/HLA interaction and are thus competent (bottom left). By contrast, in individuals that do not express the cognate HLA ligand (missing KIR ligands), NK cells cannot be educated and are hyporesponsive (bottom right).

NK cells were selectively inhibited by HLA ligands expressed on NK targets. Tarek and colleagues thus demonstrate that KIRmediated inhibition upon interaction with its HLA ligand on target cells overcomes the responsive advantage delivered during the education process (15). These observations are consistent with a previous study in the mouse showing that uneducated NK cells are critical for the response against mouse cytomegalovirus (17). Taken together, these studies reveal that during the course of cancer or viral infection, uneducated NK cells can have a dominant and beneficial impact, challenging the association of the absence of education and hyporeactivity. Thus, the calibration of NK cell reactivity resulting from MHC-I-dependent education is sufficient to prevent NK cell autoreactivity at steady state, but it can be overridden in stress conditions (tumors, microbial infections) and upon treatment with therapeutic antibodies.

\section{Could educated NK cells be rendered} as efficient as uneducated NK cells?

Several strategies can be envisioned to boost NK cell reactivity against tumors in patients. The use of therapeutic therapies combining a tumor-targeting antibody with a second antibody specifically activat- ing NK cells should be of particular interest. One way to stimulate effector NK cells is the use of anti-CD137 (4-1BB) mAb, which has shown promising ADCC boost when used in combination with rituximab (anti-CD20) or trastuzumab (anti-HER2) $(18,19)$. In NB, the persistent expression of the self-MHC-I on tumor cells is a key factor impairing the full activation of NK cells expressing self-MHC-I-specific inhibitory receptors. Thus, another promising approach to prevent the MHC-I-mediated inhibition of educated NK cells is to block inhibitory KIRs with a mAb (20). Coupling the anti-GD2 and anti-KIR mAb treatment is an attractive possibility to activate the entire NK cell population and to improve the overall survival of patients with NB and notably of those in which all KIR ligands are present.

\section{Perspectives}

Observations by Tarek and colleagues can be seen from two different perspectives. From the classical target cell recognition point of view, their findings are consistent with the inhibitory role played by KIRs, i.e., NK cells are more potent when relieved of MHC-I-dependent inhibition. However, from the education point of view, NK cells expressing KIRs which recognize MHC-I ligand are educated and are expected to display greater reactivity than uneducated NK cells. Tarek and colleagues demonstrated that in given stimulatory conditions the KIR-mediated inhibition is a strong enough signal to prevail over the responsive advantage delivered during the education process. That uneducated NK cells are not permanently hyporesponsive and can dominate antitumor responses supports the development of strategies targeting educated NK cells, such as the anti-KIR $\mathrm{mAb}$, and suggests that impacting on the education process of NK cells might not be necessarily prohibitive.

\section{Acknowledgments}

We thank the European Research Council, the Agence Nationale de la Recherche, the Ligue Nationale contre le Cancer (Equipe labellisée La Ligue), the Axa Research Fund, INSERM, CNRS, and Aix-Marseille Université for financial support to the Centre d'Immunologie de Marseille-Luminy.

Address correspondence to: Eric Vivier, Centre d'Immunologie de Marseille-Luminy, Campus de Luminy, Case 906, 13288 Marseille cedex 09, France. Phone: 33.4. 91.26.94.12; Fax: 33.4.91.26.94.30; E-mail: vivier@ciml.univ-mrs.fr. 
1. Vivier E, et al. Innate or adaptive immunity? The example of natural killer cells. Science. 2011; 331(6013):44-49.

2. Raulet DH, Vance RE. Self-tolerance of natural killer cells. Nat Rev Immunol. 2006;6(7):520-531.

3. Yokoyama WM, Kim S. How do natural killer cells find self to achieve tolerance? Immunity. 2006 24(3):249-257.

4. Brodin P, Hoglund P. Beyond licensing and disarm ing: a quantitative view on NK-cell education. EurJ Immunol. 2008;38(11):2934-2937.

5. Orr MT, Lanier LL. Natural killer cell education and tolerance. Cell. 2010;142(6):847-856

6. Bix M, Liao N-S, Zijlstra M, Loring J, Jaenisch R, Raulet D. Rejection of class I MHC-deficient haemopoietic cells by irradiated MHC-matched mice. Nature. 1991;349(6307):329-331.

7. Liao N-S, Bix M, Zilstra M, Jaenish R, Raulet D. MHC class I deficiency: susceptibility to natural killer (NK) cells and impaired NK activity. Science. 1991;253(5016):199-202.

8. Hoglund P, et al. Recognition of beta 2-microglobulin-negative (beta $2 \mathrm{~m}$-) T-cell blasts by natural killer cells from normal but not from beta $2 \mathrm{~m}$ mice: nonresponsiveness controlled by beta $2 \mathrm{~m}$ bone marrow in chimeric mice. Proc Natl Acad Sci US A. 1991;88(22):10332-10336.

9. Kim S, et al. Licensing of natural killer cells by host major histocompatibility complex class I molecules. Nature. 2005;436(7051):709-713.

10. Fernandez NC, Treiner E, Vance RE, Jamieson AM, Lemieux S, Raulet DH. A subset of natural killer cells achieves self-tolerance without expressing inhibitory receptors specific for self-MHC molecules. Blood. 2005;105(11):4416-4423.

11. Anfossi N, et al. Human NK cell education by inhibitory receptors for MHC class I. Immunity. 2006; 25(2):331-342

12. Guia $S$, et al. Confinement of activating receptors at the plasma membrane controls natural killer cell tolerance. Sci Signal. 2011;4(167):ra21.

13. Elliott JM, Wahle JA, Yokoyama WM. MHC class Ideficient natural killer cells acquire a licensed phenotype after transfer into an MHC class I-sufficient environment. J Exp Med. 2010;207(10):2073-2079.

14. Joncker NT, Shifrin N, Delebecque F, Raulet DH.
Mature natural killer cells reset their responsiveness when exposed to an altered MHC environment. J Exp Med. 2010;207(10):2065-2072.

15. Tarek N, et al. Unlicensed NK cells target neuroblastoma following anti-GD2 antibody treatment. J Clin Invest. 2012;122(9):3260-3270.

16. Parham P. MHC class I molecules and KIRs in human history, health and survival. Nat Rev Immunol. 2005;5(3):201-214.

17. Orr MT, Murphy WJ, Lanier LL. 'Unlicensed' natural killer cells dominate the response to cytomegalovirus infection. Nat Immunol. 2010;11(4):321-327.

18. Kohrt HE, et al. CD137 stimulation enhances the antilymphoma activity of anti-CD20 antibodies. Blood. 2011;117(8):2423-2432.

19. Kohrt HE, et al. Stimulation of natural killer cells with a CD137-specific antibody enhances trastuzumab efficacy in xenotransplant models of breast cancer. J Clin Invest. 2012;122(3):1066-1075

20. Romagne F, et al. Pre-clinical characterization of 1-7F9, a novel human anti-KIR therapeutic antibody that augments NK-mediated killing of tumor cells. Blood. 2009;114(13):2667-2677.

\title{
NPARM in PHOX2B: why some things just should not be expanded
}

\author{
Michael D. Gershon
}

Department of Pathology and Cell Biology, Columbia University, New York, New York, USA.

\begin{abstract}
Although the neural crest and its derivatives have been studied for a very long time, disorders of derivatives of the crest, the neurocristopathies, are not well understood. In this issue of the JCI, Nagashimada et al. provide an elegant analysis of one neurocristopathy, the association of neuroblastoma (NB) with Hirschsprung disease (HSCR) (aganglionosis of the terminal bowel) and congenital central hypoventilation syndrome (CCHS) (also known as NB-HSCR-CCHS), linked to mutations in PHOX2B. In a mouse model, Nagashimada et al. demonstrate that a disease-linked mutation promotes tumorigenesis and disrupts neurogenesis, sympathetic gangliogenesis, and crest cell colonization of the terminal bowel. They also show that mutant PHOX2B results in decreased proliferation of crest-derived cells and the development of glia at the expense of neurons. The work raises intriguing issues about the possible common origin of sympathetic and enteric nervous systems and provides new hope that we may someday understand the vexing abnormalities in gastrointestinal function that persist after the surgical treatment of HSCR.
\end{abstract}

The neural crest has long been recognized to be a gift that evolution has given to developmental biologists. It is a transient structure comprising cells that are probably heterogeneous (1), but that, as a unit, is multipotent, giving rise to melanocytes, Schwann cells, sympathetic neurons, parasympathetic neurons, enteric neurons,

Conflict of interest: The author has declared that no conflict of interest exists.

Citation for this article: $J$ Clin Invest. 2012; 122(9):3056-3058. doi:10.1172/JCI63884. enteric glia, endocrine cells, fibroblasts, muscle, bone, cartilage, and meninges (2). The neural crest thus provides opportunities to study the cellular and molecular mechanisms of epithelial-mesenchymal transformation (and the reverse), migration, aggregation, and differentiation. It has been a gift that just keeps on giving. Despite the developmental biological treasure that the neural crest has provided, however, the disorders that stem from neural crest dysfunction, the neurocristopathies (3), remain vexatious.
In this issue, Nagashimada et al. (4), in a truly remarkable study, have now provided a real insight into how a mutation in $P H O X 2 B$, a gene that encodes a paired homeodomain transcription factor that plays a critical role in the development of crest-derived autonomic neurons (5), can act in different cells in a gain-of-function or in a loss-of function manner. The gainof-function activity is tumorigenic, causing neuroblastomas (NBs) to arise in the sympathetic nervous system, while perversely synergizing with the loss-of-function effect to disrupt neurogenesis, sympathetic gangliogenesis, and crest cell colonization of the terminal bowel, which becomes aganglionic (Hirschsprung disease [HSCR]). The faults in formation of neurons and ganglia appear to be the result of a failure of the reciprocal inactivation of $\mathrm{PHOX} 2 \mathrm{~B}$ and SOX10.

During normal development, crestderived precursors of sympathetic and enteric neurons initially express SOX10, but acquire PHOX2B when they enter the preaortic (6) or enteric mesenchyme (7-9). Bipotent progenitors express both PHOX2B and SOX10, but SOX10 is inactivated in cells that are destined to form neurons, and PHOX2B is inactivated in 